\title{
Imaging of Mixed and Radiopaque Jaw Lesions
}

\author{
Filip M. Vanhoenacker, MD, PhD ${ }^{1,2,3}$ Frederik Bosmans, MD ${ }^{1,2} \quad$ Charlotte Vanhoenacker, MD 4
}

Anja Bernaerts, $\mathrm{MD}^{5}$

1 Department of Radiology, Antwerp University Hospital, Edegem, Belgium
2 Department of Radiology, AZ Sint-Maarten, Mechelen, Belgium
${ }^{3}$ Department of Radiology, Ghent University Hospital, Ghent, Belgium
${ }^{4}$ Department of Radiology, University Hospitals Leuven, Leuven, Belgium
${ }^{5}$ Department of Radiology, GZA Hospitals, Antwerp, Belgium

Address for correspondence Filip M. Vanhoenacker, MD, PhD, Department of Radiology, University Hospital of Antwerp UZA, Wilrijkstraat 10, 2650 Edegem, Belgium (e-mail: filip.vanhoenacker@telenet.be).

Semin Musculoskelet Radiol 2020;24:558-569.

\begin{abstract}
Radiopaque lesions and lesions of mixed density are less frequent than radiolucent lesions of the jawbones. They comprise a spectrum of odontogenic and non-odontogenic lesions. The latter group includes inherited and developmental disorders, osteomyelitis, and benign and

Keywords

- jaws

- tumor and tumor-like lesions

- increased bone density

- cone beam computed tomography

- imaging malignant primary bone tumors and metastases. Most odontogenic radiopaque or mixed lesions are either related to the apex or more rarely to the crown of the tooth, although there are exceptions to this rule. Some lesions, such as a torus mandibularis and torus palatinus, have a characteristic location, whereas others show no relationship to the dentition. This article describes the most characteristic and prevalent radiopaque and mixed lesions of the jaws and their imaging characteristics. Paget's disease, fibrous dysplasia, and rare sclerotic bone diseases of the maxillofacial bones are discussed elsewhere in this issue. Careful correlation of clinical presentation, panoramic radiographs, cone beam computed tomography, and histopathology are the cornerstones for appropriate lesion characterization.
\end{abstract}

\section{Lesions with Preferential Periapical Location}

\section{Cementoblastoma}

Cementoblastoma is a rare neoplasm of cementoblasts around the apex of a tooth root. ${ }^{1}$ The mandible is more frequently involved than the maxilla with predilection of the premolar or first molar of the mandible., ${ }^{2,3}$ Children and adults $<30$ years are usually affected. ${ }^{1,2}$

On panoramic radiographs and cone beam computed tomography (CBCT), the lesion appears as a well-delineated round radiopaque mass of disorganized cementum often surrounded by a thin radiolucent halo of nonmineralized germinative cells. ${ }^{2}$ Cementoblastoma typically fuse with the root of one or more teeth and may invade the root canal and pulp chamber. ${ }^{4}$ Because of the intimate relationship with the root of the teeth, tooth extraction may be difficult. ${ }^{3}$

\section{Cemento-ossifying Fibroma}

Cemento-ossifying fibroma is a benign neoplasm composed of fibrous tissue and mineralized material, often found in the third and fourth decades with a marked female predilec- tion. ${ }^{5,6}$ Clinical presentation consists of a painless slowgrowing mass that may cause facial asymmetry.

On panoramic radiographs and CBCT (- Fig. 1 and -Fig. S1), the lesion is usually mixed radiopaque-radiolucent and less commonly either radiolucent or radiopaque. Advanced lesions become more radiopaque due to progressive matrix mineralization. The lesion is well delineated and grows concentrically with buccolingual expansion. Large lesions may cause tooth displacement and root resorption. ${ }^{7}$ The mandibular canal may be displaced inferiorly, whereas in fibrous dysplasia the canal may be displaced in any direction. ${ }^{8,9}$

\section{Periapical Cemento-osseous Dysplasia}

Although its precise etiology has not been unraveled, periapical cemento-osseous dysplasia (PCOD) is believed to be a reactive process resulting from proliferation of connective tissue within the periodontal membrane. ${ }^{2,3}$ PCOD typically presents as an asymptomatic lesion in the bone around the root apices of the mandibular incisors of middle-aged black women. Some lesions can be painful. ${ }^{3}$
Issue Theme Facial and Dental Musculoskeletal Imaging; Guest Editor, Filip M. Vanhoenacker, MD, PhD
Copyright $\odot 2020$ by Thieme Medical Publishers, Inc., 333 Seventh Avenue, New York, NY 10001, USA. Tel: +1(212) 584-4662.
DOI https://doi.org/ 10.1055/s-0039-3402766. ISSN 1089-7860. 

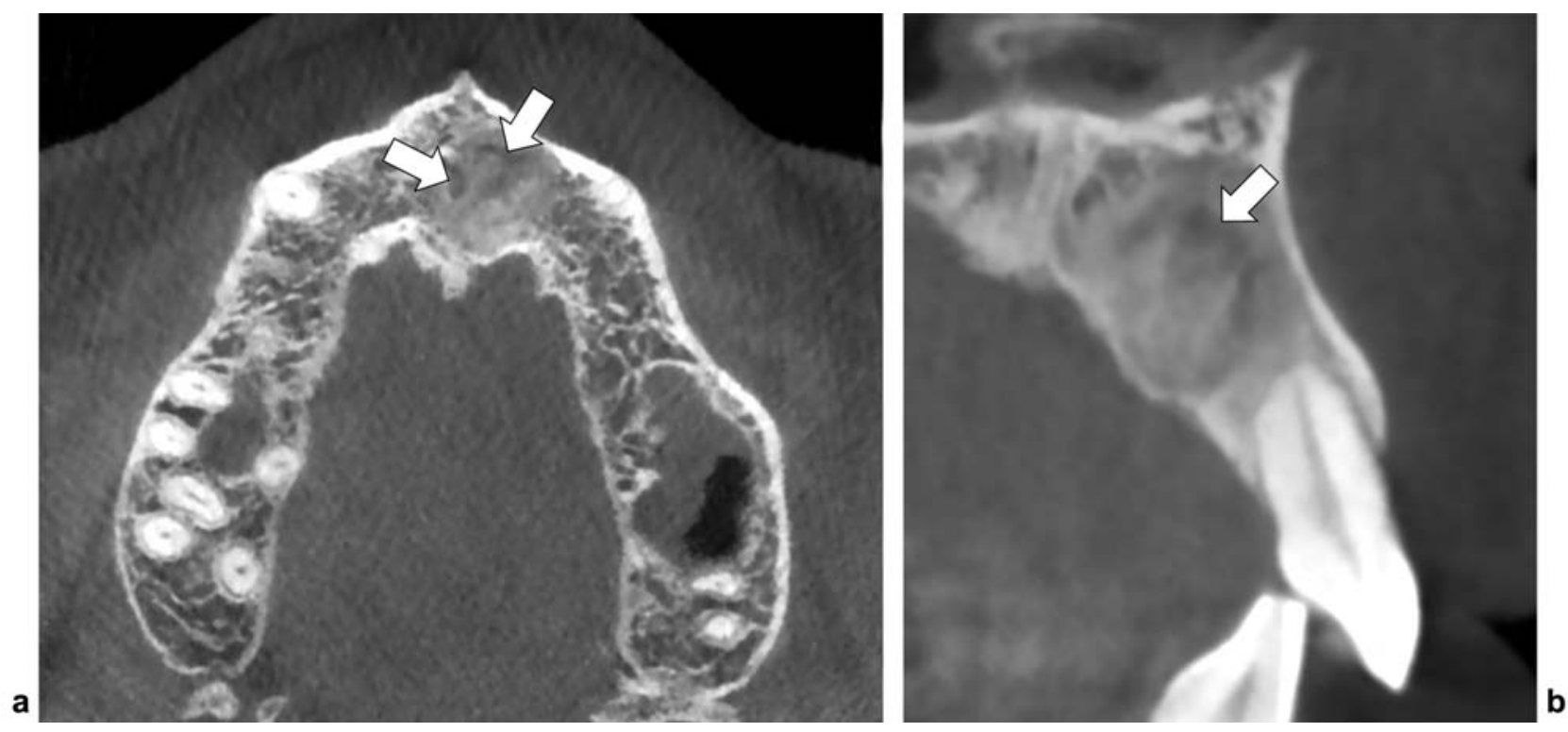

Fig. 1 Cemento-ossifying fibroma of the maxilla. (a) Axial and (b) sagittal reformatted CBCT images show a well-delineated radiopaque lesion with ground-glass appearance in the left anterior maxilla. Note some intralesional radiolucent foci (white arrows) and resorption of the root of tooth 22.

Radiographically, three stages may be seen depending on the age of the lesions: initially, the lesion is well defined and radiolucent (osteolytic stage), followed by a mixed radiolucentradiopaque stage ( - Fig. $\mathbf{2}$ ), with a dense central core (cementoblastic stage), and finally a mineralized radiopaque mass surrounded by a thin radiolucent zone may develop (mature inactive stage). The tooth remains vital in contradistinction to the devitalized tooth seen with periapical granuloma. ${ }^{10}$

The term florid cemento-osseous dysplasia (FCOD) is used for a massive involvement of periapical cemental dysplasia involving two of four quadrants of the tooth-bearing areas of the jawbones. ${ }^{2,11,12}$ It is most frequently seen in the mandible of middle-aged black women, although it may also occur in whites and Asians. ${ }^{1,3}$ The lesion is found incidentally during routine dental examination and is often asymptomatic or may present with pain. ${ }^{11}$

On radiographs and $C B C T$, FCOD presents as multifocal mixed osseous changes in the jawbones. ${ }^{2}$ There is often bone expansion. Sclerotic lesions do not fuse with the tooth root and are located above the mandibular canal. ${ }^{13}$
FCOD usually has no hereditary basis, but familial cases have been reported. Familial FCOD has no demographic predilection, presents at a younger age, and may progress more rapidly than its sporadic counterpart. ${ }^{11}$

The differential diagnosis on imaging includes fibrous dysplasia, Paget's disease, Gardner's syndrome, chronic sclerosing osteomyelitis, and osteoblastic bone metastasis. ${ }^{11,12}$

Fibrous dysplasia (FD) is often polyostotic and involves the maxilla more frequently than the mandible. ${ }^{11}$ It is typically unilateral and often extends into contiguous bones such as the zygoma and sphenoid. ${ }^{11}$ FD may be radiolucent in the initial stage but typically has a ground-glass appearance and may cause superior displacement of the mandibular canal. 3,14

Paget's disease is also often polyostotic but rather involves the entire mandible and is characterized by elevated alkaline phosphate levels, whereas laboratory findings in FCOD are normal. ${ }^{12}$

Gardner's syndrome is not restricted to the tooth-bearing area and may involve other bones than the jawbones. ${ }^{11}$
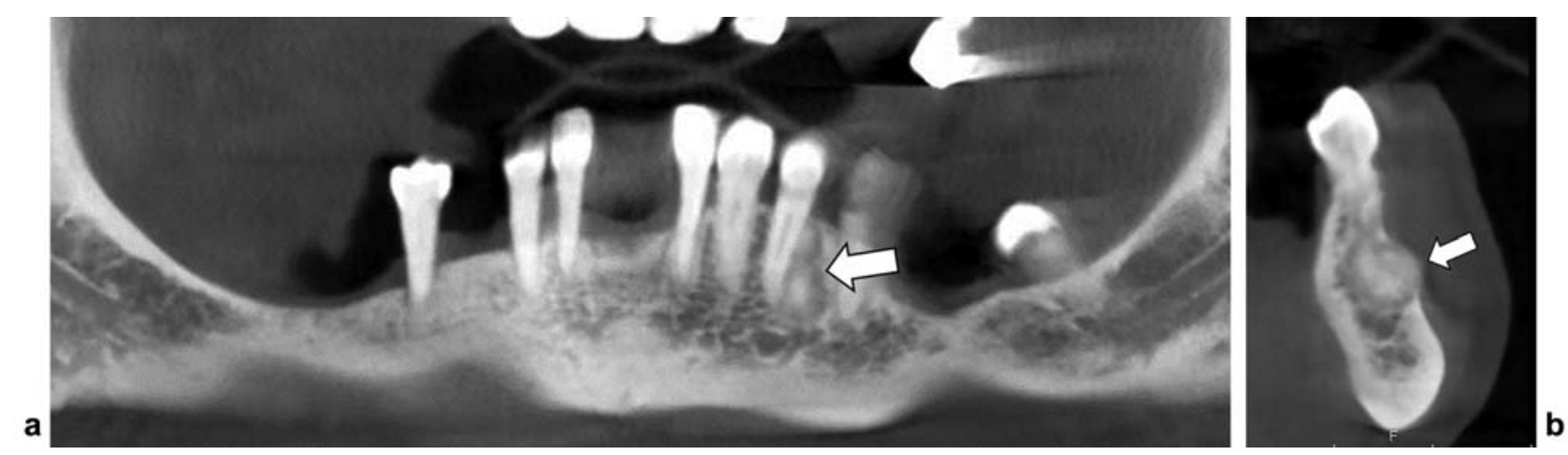

Fig. 2 Periapical cemento-osseous dysplasia, cementoblastic type. (a) Panoramic and (b) sagittal reformatted CBCT images show a mixed radiolucent-radiopaque lesion around the apex of the left mandibular canine at the left anterior mandible with a dense central core (arrow). 

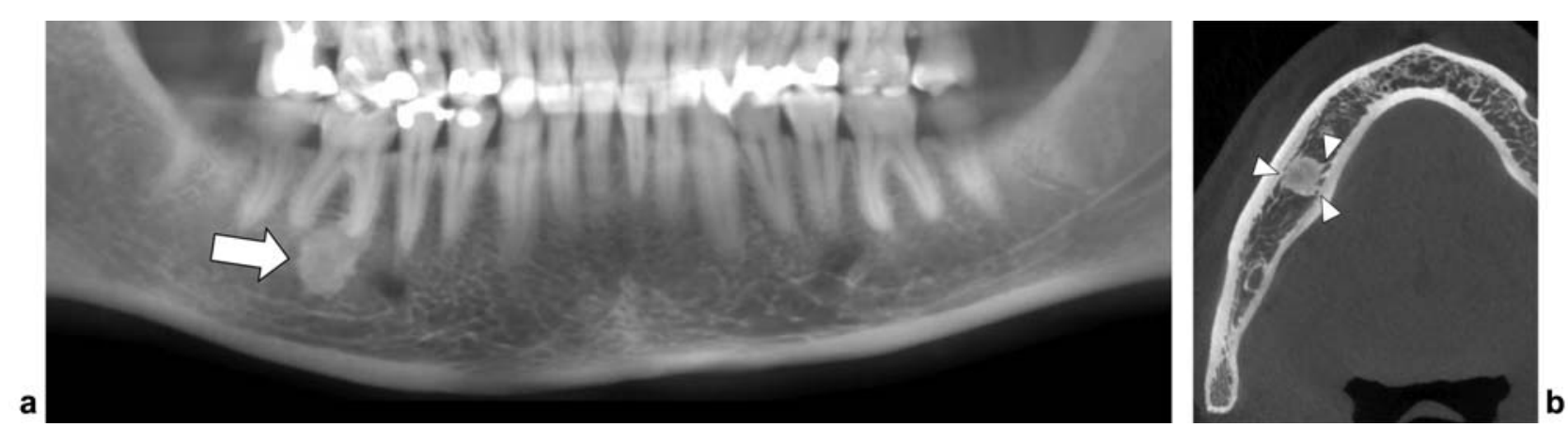

Fig. 3 Condensing osteitis. (a) Panoramic reformatted CBCT image demonstrates a radiopaque lesion adjacent to the apex of tooth 46 . The lesion has spicular borders (arrow). (b) Axial reformatted CBCT image shows blending of the spicular borders with the adjacent trabeculae (arrowheads).

In chronic osteomyelitis, usually one bone segment is involved and the lesion is poorly circumscribed, whereas FCOD is seen as multiple round or lobulated opaque masses. Chronic osteomyelitis usually involves the body of the mandible from the alveolus to the inferior border and may extend into the ramus. ${ }^{12}$ However, due to a decreased vascularity of the lesion, FCOD may predispose to chronic infection and pathologic fracture with poor healing. ${ }^{11}$

Asymptomatic FCOD needs no surgical treatment. A waitand see policy is recommended with imaging follow-up every 2 to 3 years. ${ }^{11}$

\section{Condensing Osteitis}

Condensing osteitis, also known as focal sclerosing osteomyelitis, represents a mild focal form of sclerosing osteomyelitis arising in the posterior mandible at the apices of the premolar or molar teeth. ${ }^{15}$ It is the most common sclerotic lesion of the jaw and usually affects children and young adults. ${ }^{13}$

Radiologically or on CBCT ( - Fig. 3 ), it appears as a focal area of well-circumscribed or ill-defined bone sclerosis surrounding the dental roots, often with inflamed or necrotic dental pulp. ${ }^{11}$ The lesion may be single or multiple. ${ }^{4}$

\section{Idiopathic Osteosclerosis}

This incidental lesion presents radiographically as a radiopaque area adjacent to the tooth apex or interradicular region, most commonly in the premolar or molar region. It occurs in young asymptomatic patients and may be multifocal.

Radiographically or on CBCT ( - Fig. 4), the lesion has sharp angular margins. ${ }^{13}$ This lesion resembles a dense bone island, but in the latter, there is no specific relationship with the dentition. A typical dense bone island has spiculated margins.

\section{Lesions with Preferential Pericoronal Location}

\section{Odontoma}

Odontomas are hamartomas originating from differentiated epithelial and mesenchymal cells (ameloblasts and odontoblasts) and composed of enamel and dentin. ${ }^{16,17}$ Odontomas are the most common odontogenic neoplasms. They are most prevalent in children and young adults. ${ }^{2,15}$

There are two macroscopic types. The compound odontoma consists of multiple denticles or small teeth-like struc- tures. The complex odontoma does not resemble a normal tooth and appears as an amorphous calcified mass. ${ }^{17}$

Radiographically, the compound type ( - Fig. 5 and -Fig. S2) consists of multiple small radiopaque teeth-like structures surrounded by a thin radiolucent halo. The complex odontoma (-Fig. 6) presents as an irregularly shaped and amorphous radiopaque mass, also surrounded by a thin radiolucent halo. ${ }^{18}$

The size of the lesions may range between 1 and $3 \mathrm{~cm} .{ }^{19}$ The posterior mandible and ramus is most commonly involved in complex odontoma, whereas a compound odontoma is most common in the anterior maxilla. ${ }^{3,4,15}$ Lesions may have a mass effect causing impaction, malpositioning, and resorption of adjacent teeth. ${ }^{1,2,20}$

Surgical excision is the treatment of choice. Recurrence is rare. $^{19}$

\section{Adenomatoid Odontogenic Tumor}

This adenomatoid odontogenic tumor was formerly known as odonto-ameloblastoma. ${ }^{21}$ It may be located around the crown of the tooth or without any relation to the tooth. It is most frequently located in the anterior maxilla in the second decade of life and predominantly in female patients. ${ }^{2}$

Radiographically and on CBCT, it is primarily of mixed density consisting of a well-delineated radiolucent lesion with intralesional flecks of calcifications with a "faint snowflake" or "small pebble" appearance. Most of the lesions are associated with an impacted tooth that is completely contained within the lesion, in contrast with a dentigerous cyst that typically attaches at the cementoenamel junction. ${ }^{2}$

\section{Calcifying Epithelial Odontogenic Tumor}

Calcifying epithelial odontogenic tumor (CEOT), or Pindborg tumor, is a rare odontogenic neoplasm originating from odontogenic epithelium. It can occur at any age, but it is most common in the fifth decade. The posterior mandible is most often involved with predilection of the first and second molars. ${ }^{10,22,23}$ It may be located either around the crown of the tooth or is not tooth related. ${ }^{2}$

On radiographs and $\mathrm{CBCT}$, the lesion has a variable density ranging from radiolucent, mixed radiolucent-radiopaque, to dense radiopaque. ${ }^{24}$ Clustered intralesional calcifications are typically seen around the tooth crown, and the lesion is often associated with impaction of one or multiple teeth. ${ }^{24}$ CEOT 
a
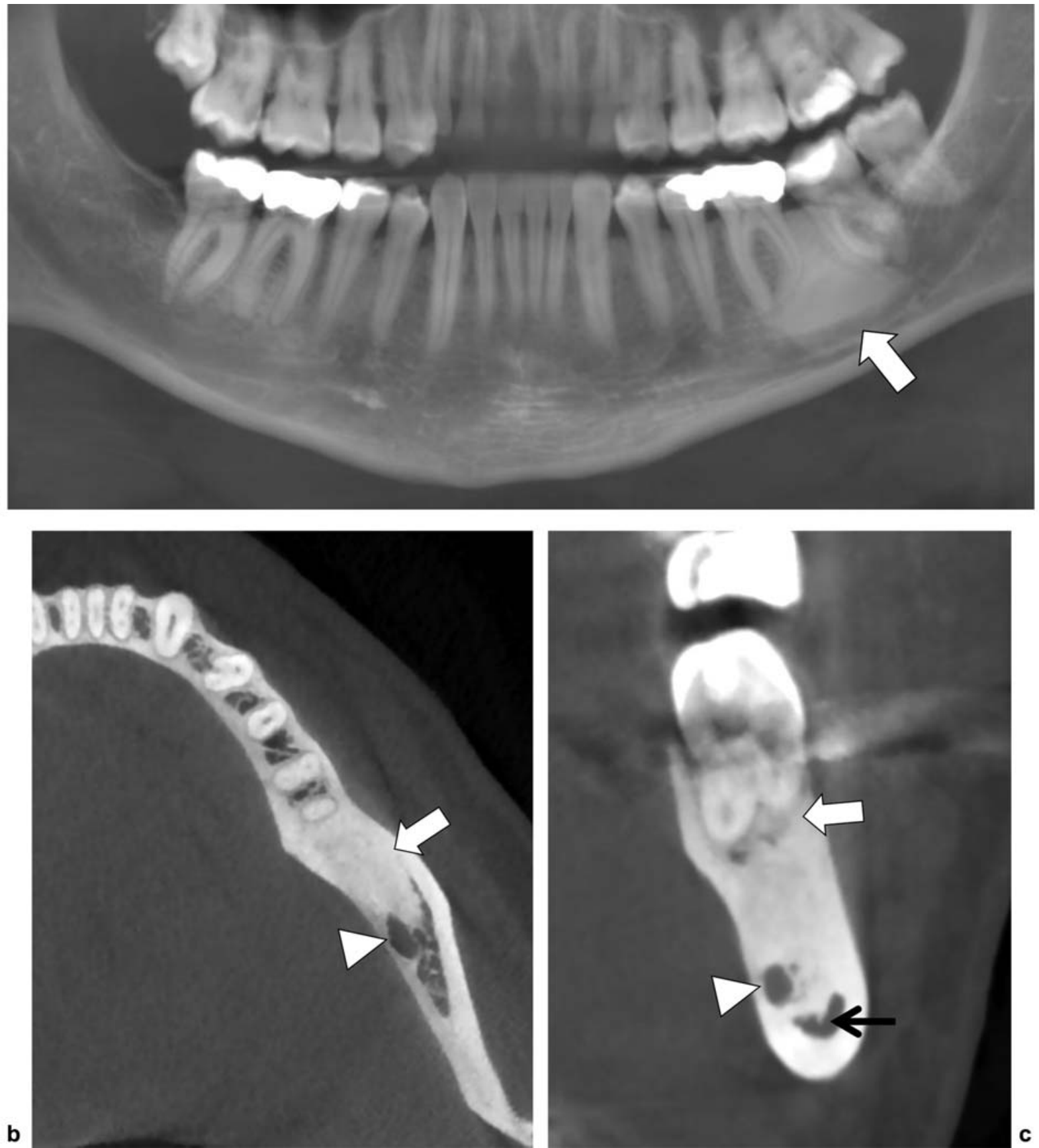

Fig. 4 Idiopathic osteosclerosis. (a) Panoramic reformatted CBCT image showing a well-defined radiopaque lesion in the left mandible between the roots of tooth 36 and 37 (arrow). There is root displacement of 36 and 37. (b) Axial reformatted CBCT image confirms the radiopaque lesion in the left mandible (arrow). There is an intimate relationship with the mandibular canal (arrowhead). (c) Sagittal reformatted CBCT image confirms the intimate relationship with the mandibular canal (arrowhead) and the dental roots (arrow). The lesion blends into the buccal and lingual cortex. Note some spiculation at the inferior aspect of the lesion (thin arrow).

may be either well defined or ill-defined and may have a unilocular or multilocular appearance.

\section{Dentinogenic Ghost Cell Tumor}

Dentinogenic ghost cell tumor (DGCT) is considered a rare neoplastic variant of CEOT. ${ }^{25}$ There are two distinct types of DGCT. The intraosseous DGCT tends to be locally aggressive with a mean age of 40 years. It mainly occurs in the canine to first molar region, usually presents as a painless bony swelling, although slight numbness and pain may occur as well. The extraosseous DGCT, in contrast, exhibits limited growth potential and usually occurs in the sixth decade. ${ }^{26}$ The anterior regions of the jawbones are usually affected, typically at edentulous areas. Clinically, a firm painless nodule on the gingival or alveolar mucosa is seen. ${ }^{27}$

Radiographically and on CBCT, a variable appearance may be seen. Although the lesion is most often of mixed density with a variable degree of calcification, it can also present as a completely radiolucent lesion. Most cases are unilocular, but multilocular lesions may be observed as well. These tumors 

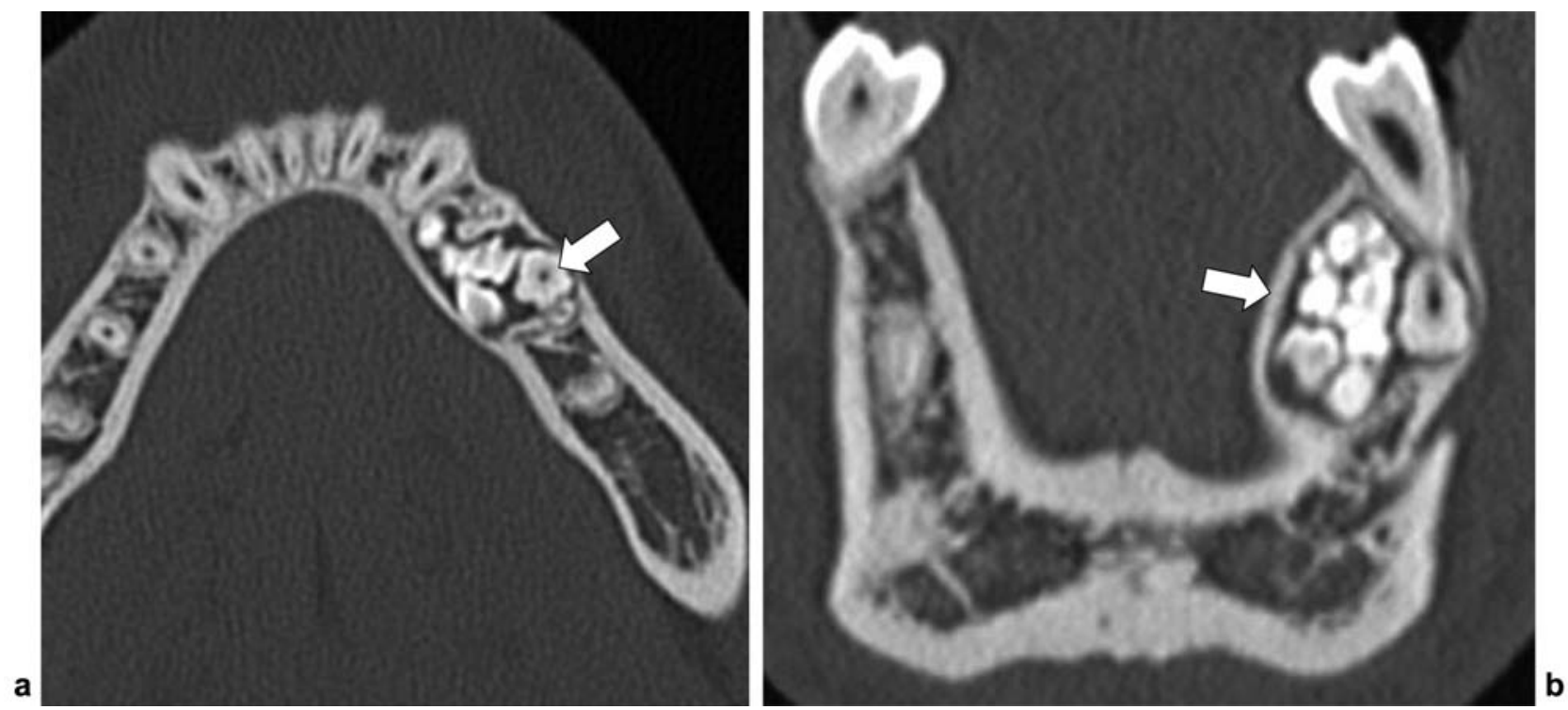

Fig. 5 Compound odontoma. (a) Axial and (b) coronal reformatted dental CT images show multiple small radiopaque tooth-like structures at the left anterior hemimandible with surrounding radiolucent halo (arrows).

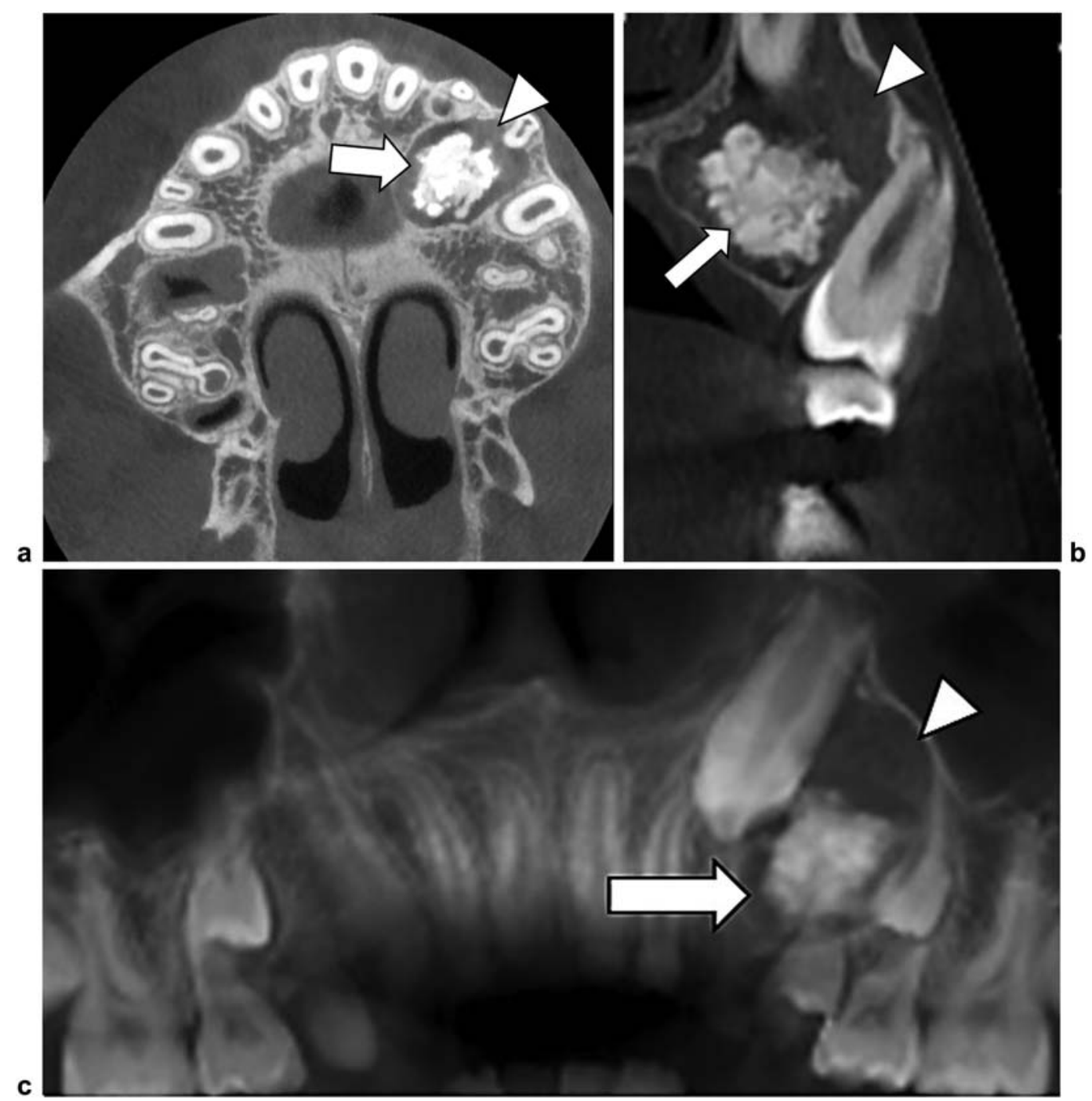

Fig. 6 Complex odontoma in an 11-year-old patient. (a) Axial image, (b) parasagittal reformatted CBCT images, and (c) panoramic image show an amorphous radiopaque lesion within the left upper jaw around the crown of tooth 24 (arrows), surrounded by a lucent follicle (arrowheads) formed by a fibrous capsule. 


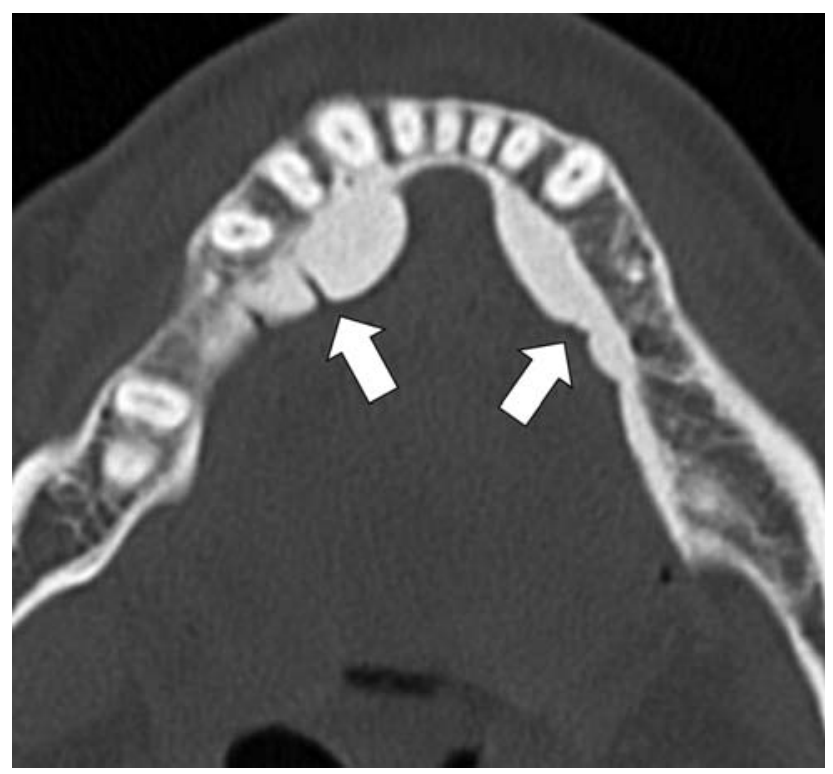

Fig. 7 Torus mandibularis. Axial reformatted dental CT image shows a lobulated radiopaque outgrowth at the lingual aspect of the right and left mandible (arrows).

are typically well defined, often expansile, and may result in resorption and divergence of roots of adjacent teeth. ${ }^{27}$

\section{Lesions with a Specific Location}

A torus is a localized outgrowth of cortical bone arising at a specific location of the mandible or maxilla. The pathogenesis is unknown. ${ }^{13}$ Tori are usually asymptomatic or may interfere with fitting of a dental prosthesis. They may become symptomatic following trauma. ${ }^{4}$

\section{Torus Mandibularis}

A torus mandibularis ( $\mathbf{- F i g . ~} \mathbf{7}$ and $\boldsymbol{- \text { Fig. }}$. 3 ) typically arises from the lingual surface of the mandible above the origin of the mylohyoid muscle. The lesion is usually bilateral and may vary in size and morphology, ranging from flat, nodular, or slightly pedunculated. ${ }^{2,13}$
Torus mandibularis should not be confused with genial tubercles that are prominent insertional spines at the mandibular insertion of the geniohyoid and genioglossus muscles. ${ }^{2}$

\section{Torus Palatinus}

A torus palatinus ( $\mathbf{F i g . ~} 8$ and $\mathbf{- F i g . ~} \mathbf{5 4}$ ) is a similar nodular bony overgrowth at the midline of the hard palate.,15

\section{Torus Maxillaris}

A torus maxillaris arises at the lingual surface of the posterior maxilla. $^{4}$

\section{Lesions That May Not Have Contact with Teeth}

\section{Odontoma}

Odontoma may occasionally show no contact with the teeth on imaging. For further detailed discussion of the imaging appearance of odontoma, see the earlier discussion.

\section{Bone Island}

Dense bone islands, or enostomas, are common incidental findings, consisting of failure of resorption of secondary spongiosa within the trabecular bone. ${ }^{15}$ Any bone, including the jawbones, may be affected.

Radiologically and on CBCT (-Fig. 9), the sclerotic focus typically has peripheral thorny radiations, blending with the trabeculae of the surrounding spongiosa. The shape may be round, oval, or oblong.

The lesion should not be confused with an osteoblastic metastasis. The presence of spicular borders argues against osteoblastic metastasis. In addition, attenuation measurements on CT may also be useful to distinguish untreated osteoblastic metastases from enostomas in patients with a history of a primary malignancy. A mean attenuation of 885 $\mathrm{HU}$ and a maximum attenuation of 1,060 $\mathrm{HU}$ provide reliable thresholds below which a metastatic lesion is the favored diagnosis. ${ }^{15}$ On magnetic resonance imaging (MRI), the lesion is typically of low signal on all pulse sequences. Most bone islands do not grow and do not demonstrate scintigraphic activity. ${ }^{15}$

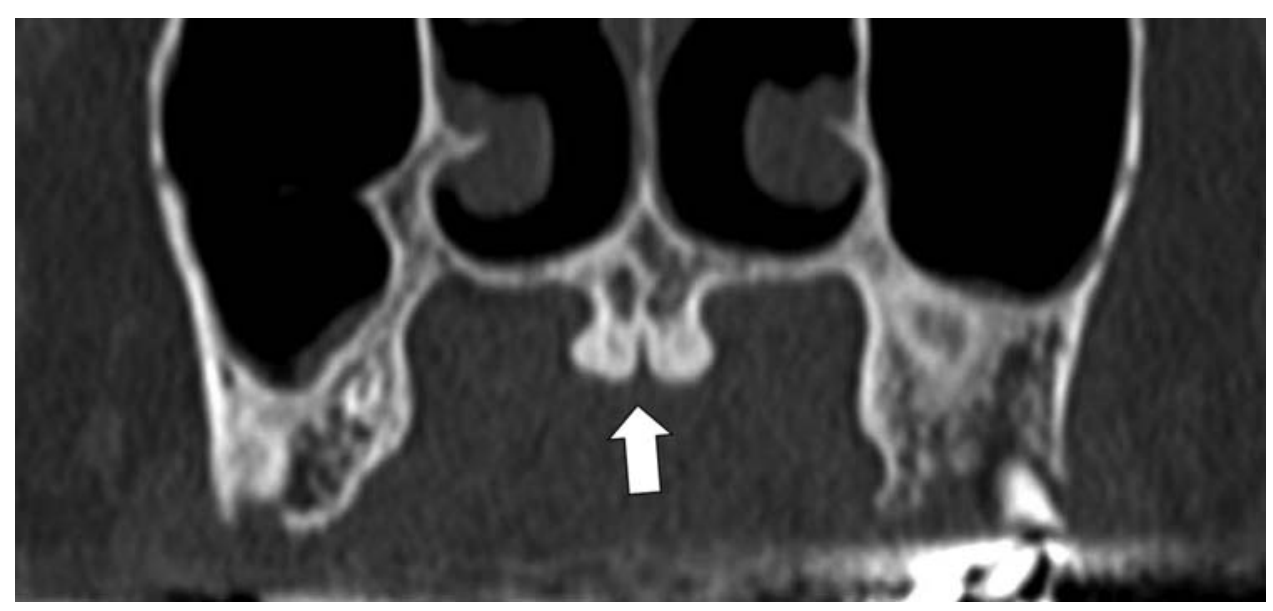

Fig. 8 Torus palatinus. Coronal reformatted dental CT image shows a paramedian bilateral radiopaque outgrowth at the hard palate (arrow). 


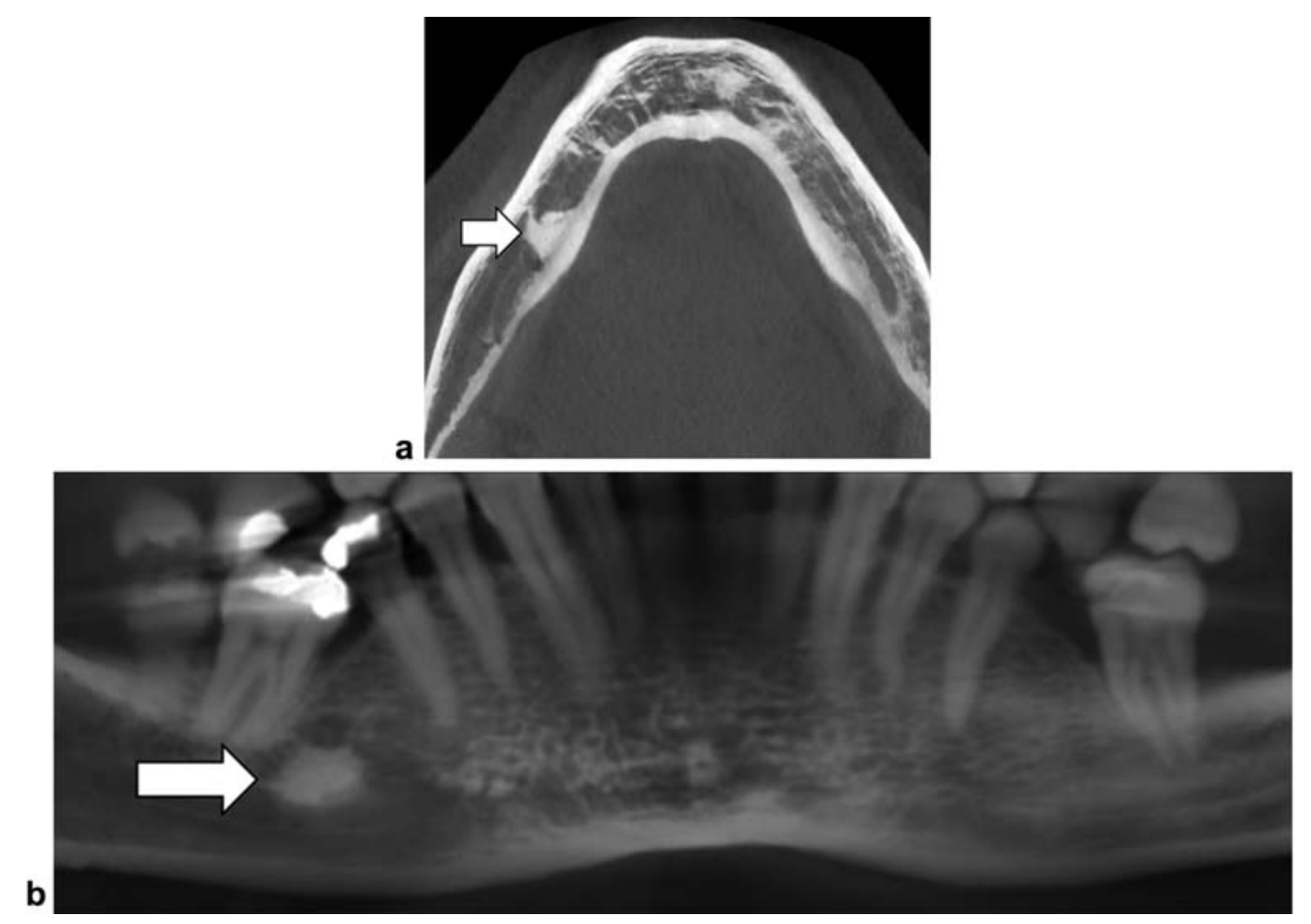

Fig. 9 Bone island. (a) Axial and (b) panoramic reformatted images showing a dense bone island with spicular margins within the right mandible (arrows). The lesion has no relationship with a tooth. It should be differentiated from sclerosing (condensing) osteitis that represents a chronic inflammation usually around the apex of a tooth. In the latter, there is often associated widening of the periodontal ligament around the tooth.

\section{Osteoma}

Osteomas are benign tumors composed of mature compact or cancellous bone and mostly arise in the craniofacial bones (-Fig. 55), with the posterior mandibular body or condyle the most common. ${ }^{4}$ Bone expansion may be seen. Multiple osteomas are associated with Gardner's syndrome. ${ }^{4}$

Osteomas are further discussed in the article "Fibrous Dysplasia, Paget's Disease, and Uncommon Bone Lesions of the Craniofacial Bones."

\section{Chronic Osteomyelitis}

Chronic osteomyelitis of the jawbones results from either a persistent infection due to inadequately treated acute infection or a long-term low-grade infection. ${ }^{2,15}$ There are three types of chronic osteomyelitis of the jaws.

The first type, chronic sclerosing osteomyelitis, has a predominant sclerotic component that may be either diffuse or focal. The focal subtype is also designated as periapical osteitis or condensing osteitis and presents radiographically and on CBCT (-Fig. S6) as a well-circumscribed periapical radiopaque lesion (see earlier). ${ }^{2,15}$ The diffuse subtype is less frequent and characterized by diffuse endosteal sclerosis on radiographs and $\mathrm{CBCT}^{2}{ }^{2}$

Chronic suppurative osteomyelitis (-Fig. 10) is the second subtype and characterized radiologically by a mixture of bone destruction, sequestrum, and extensive involucrum formation. ${ }^{2,15}$ Inflammation into the adjacent soft tissue may cause myositis, abscess formation, and sinus tracts. ${ }^{2}$

The third type is osteomyelitis with proliferative periostitis or Garré osteomyelitis (-Fig. 11). It is typically seen in children and adolescents. On radiographs and on $\mathrm{CBCT}$, there is a multilamellar periosteal reaction, with an onion peel appearance, most often located at the inferior, buccal, and lingual aspect of the mandible., ${ }^{2,15}$

\section{Osteoradionecrosis}

Osteoradionecrosis of the jawbones may develop after radiation therapy for head and neck tumors receiving a dose $>60 \mathrm{~Gy} .{ }^{15} \mathrm{It}$ usually occurs between 4 months and 3 years following radiotherapy. ${ }^{15}$ It most commonly affects the body of the mandible, with typical sparing of the chin and the angles, presumably because of its muscular insertions. ${ }^{4}$ The buccal cortex is more vulnerable than the lingual cortex. ${ }^{4}$

On radiograph and CBCT ( - Fig. 12 and - Fig. $\mathbf{5 7}$ ), osteoradionecrosis exhibits a mixed lytic and sclerotic appearance, with areas of fragmentation or sequestration. Intralesional gas may be seen as well and strongly supports the diagnosis of osteoradionecrosis. ${ }^{1,13,28,29}$

The lesion may mimic tumor recurrence. ${ }^{30}$ The presence of buccal erosions and involvement of the contralateral side of the mandible are important clues to the diagnosis of osteoradionecrosis. $^{31,32}$ Diffusion-weighted MRI may be used to differentiate tumor recurrence from osteoradionecrosis. High apparent diffusion coefficient (ADC) values are seen in necrosis, whereas low ADC values are seen in recurrent tumor. ${ }^{33}$

\section{Bisphosphonate-related Osteonecrosis}

Bisphosphonate-related osteonecrosis of the jaws (BRONJ) represents bone necrosis secondary to bisphosphonate treatment, a drug used to treat a variety of diseases including osteoporosis, multiple myeloma, metastatic disease, and 

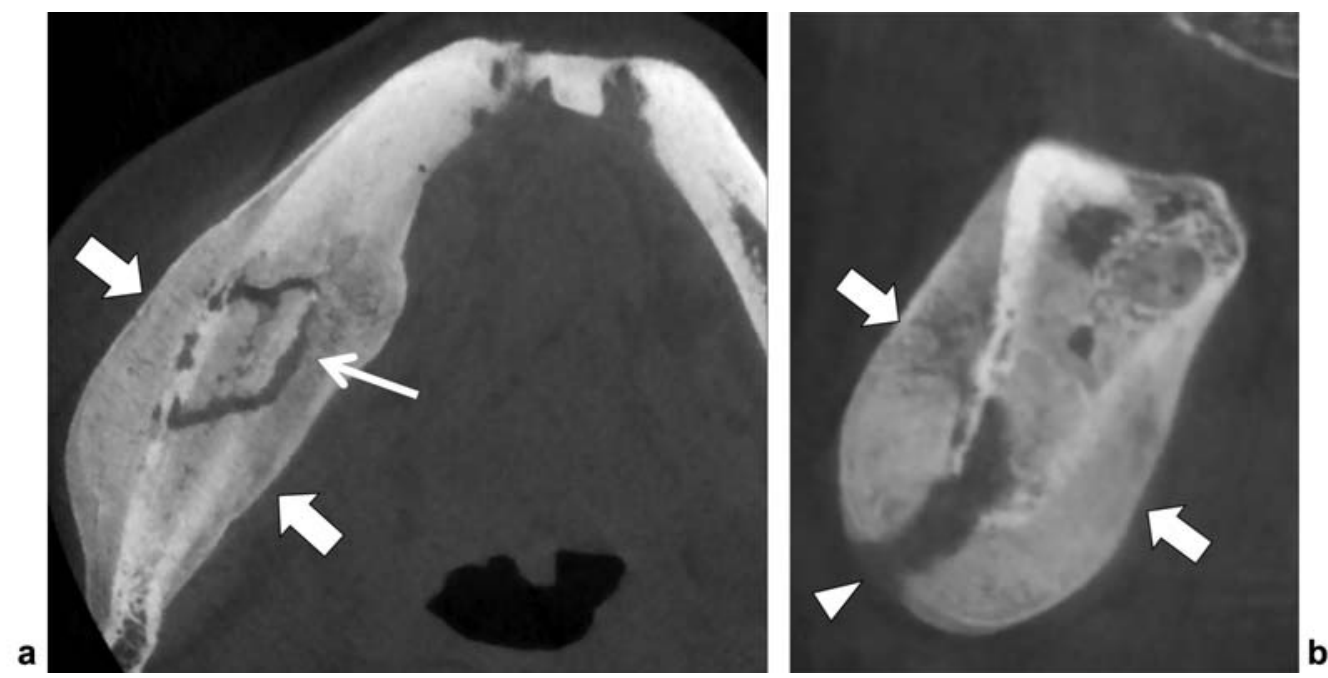

Fig. 10 Chronic suppurative osteomyelitis. (a) Axial CBCT image shows a mixture of bone destruction with intralesional sequestrum (thin arrow) and extensive periosteal reaction (involucrum) at the buccal and lingual cortex of the right mandible (arrows). (b) Sagittal reformatted CBCT image shows extensive periosteal reaction (involucrum) (arrows) and a sinus tract to the buccal cortex (arrowhead).
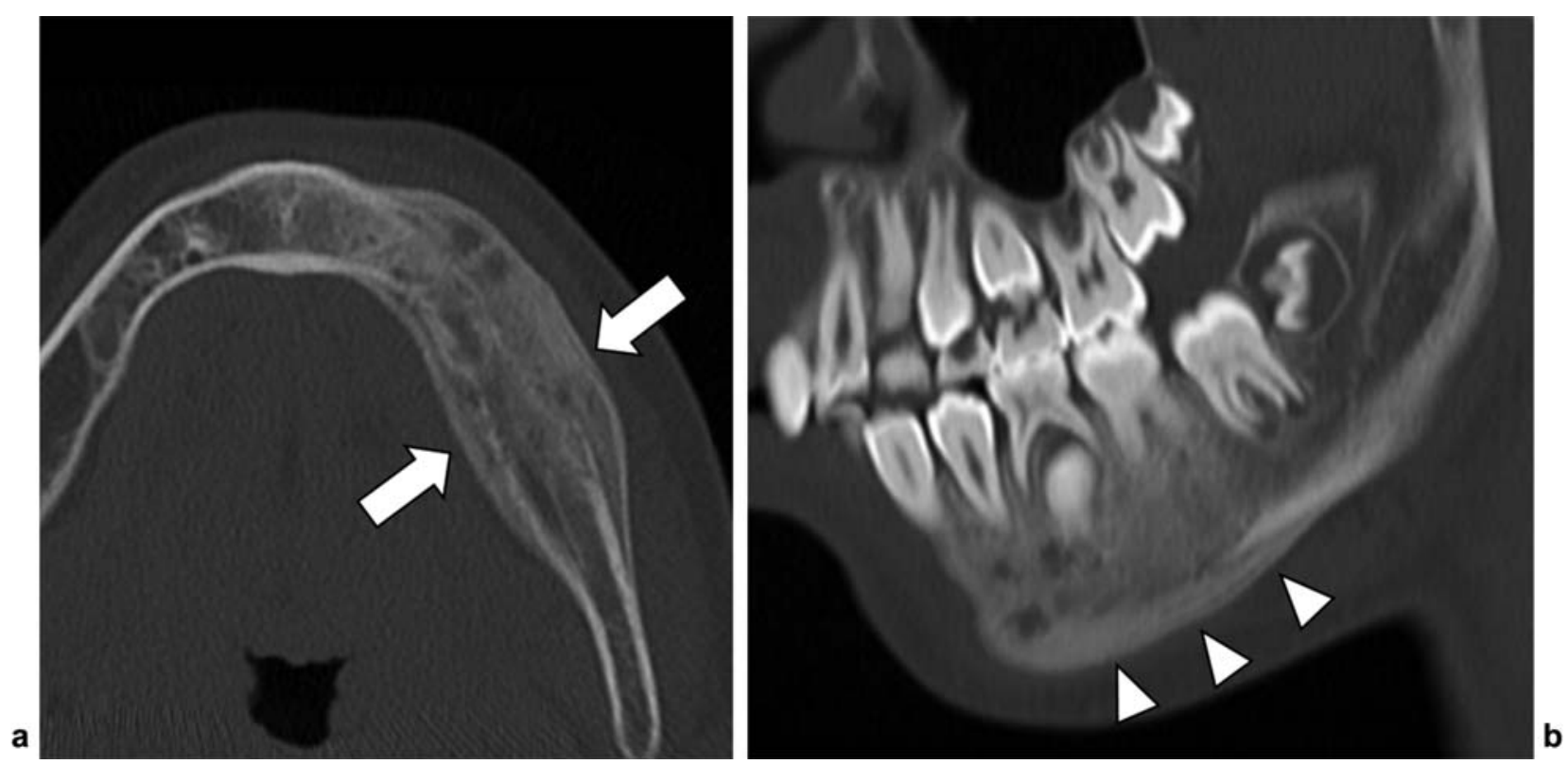

Fig. 11 Osteomyelitis with proliferative periostitis of Garré in a 12-year-old boy. (a) Axial CT image shows sclerosis of the left mandible and marked periosteal reaction of the buccal and lingual cortex (arrows). (b) Oblique sagittal reformatted CBCT image shows inhomogeneous sclerosis of the mandible (arrowhead).

Paget's disease of bone. ${ }^{13,29}$ Concurrent steroid treatment may further increase the risk for BRONJ. ${ }^{4}$

BRONJ commonly involves the mandible most often at the mylohyoid line. ${ }^{4}$ The maxilla is less frequently involved because of its richer blood supply. ${ }^{4}$ It may occur spontaneously or may be precipitated by tooth extraction or dental surgery. ${ }^{4,33}$ Clinical presentation is usually painful but may be asymptomatic as well. ${ }^{4}$

On radiographs and СBCT (-Fig. 13 and -Fig. 58), a mixture of bone sclerosis and osteolysis is seen. Periosteal reaction, fistula formation, and pathologic fracture may occur in long-standing disease. ${ }^{34}$

\section{(Osteo)chondroma}

Enchondroma originate from cartilaginous remnants in the bone. Compared with their occurrence in the long bones, they are rare in the jawbones. Radiographically and on CBCT, the lesion may be radiolucent with typical intralesional popcorn or ring-and-arc chondroid-like calcification. On MRI, they have a typical cartilaginous matrix, which is of high signal on T2weighted images and show a ring-and-arcenhancement pattern after intravenous administration of gadolinium contrast. Morphologically, the lesions have a multilobular appearance.

Osteochondroma or cartilaginous exostosis are also rarely seen at the craniofacial bones. ${ }^{35}$ These tumors have a predilection for 


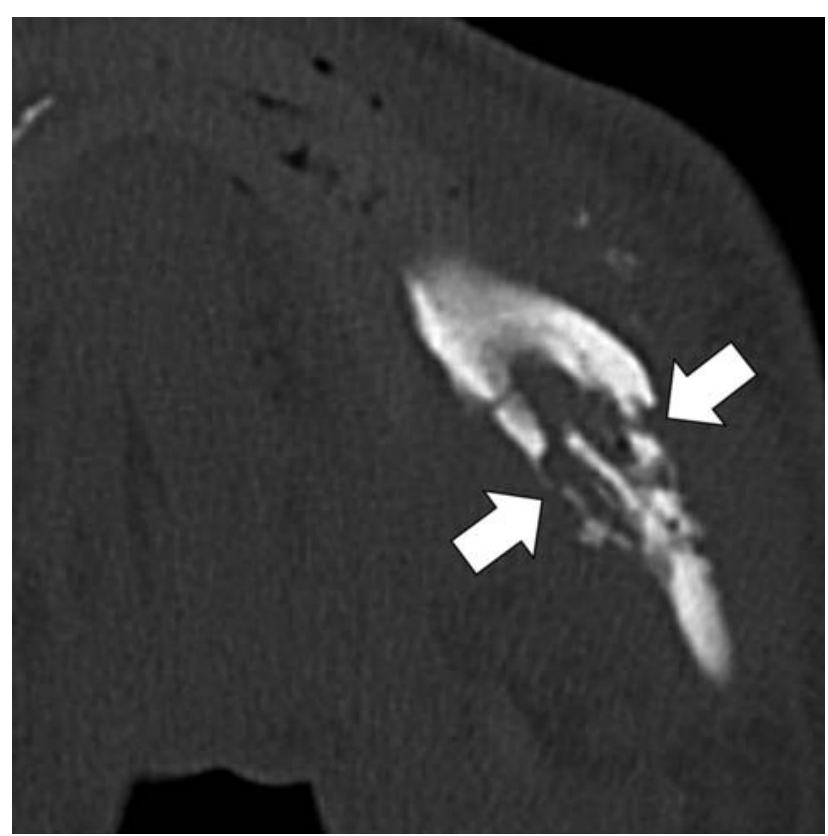

Fig. 12 Osteoradionecrosis. Axial CT image shows irregular delineated bone destruction with cortical breakthrough of the buccal and lingual cortex of the left mandible (pathologic fracture) (arrows). Note subtle intralesional gas and sequestrum formation.

the coronoid and condylar processes of the mandible ${ }^{2}$ that may exhibit a focal mushroom-shaped enlargement. Osteochondroma are usually solitary but may be part of hereditary multiple exostosis syndrome (HMES). However, involvement of the mandible has only been exceptionally reported in association with HMES. ${ }^{35,36}$
Small lesions may be asymptomatic, but larger lesions may cause a decreased mouth opening. Continuity of the cortex and the medullary bone of the lesion and the underlying host bone is a characteristic imaging finding on plain radiographs and CT (-Fig. 14). The surface is typically covered by a cartilaginous cap that is of high signal intensity on T2-weighted images. The thickness of the cartilage cap is an important parameter to differentiate benign osteochondroma from chondrosarcomas and should not exceed $2 \mathrm{~cm}$ in benign osteochondroma. If thicker, malignancy should be suspected. Although malignant degeneration is a rare occurrence in solitary osteochondroma, it is much more frequent in HMES. ${ }^{36,37}$

\section{Osteoblastic Metastasis}

Osteoblastic metastasis rarely occurs in the jawbones and is predominantly seen in prostate carcinoma, although other primary carcinomas (e.g., breast, thyroid, kidney) may also have a mixed or osteoblastic appearance. Approximately 80 to $85 \%$ of metastases involve the mandible, most commonly the molar region. ${ }^{11,38,39}$ Compared with osteosarcoma, an aggressive periosteal reaction is rare in osteoblastic metastasis ( - Fig. 15 and - Fig. S9) but may occur, resulting in a pseudosarcomatous imaging appearance.

\section{Osteosarcoma}

Osteosarcoma of the jawbones or gnathic osteosarcomas account for up to $10 \%$ of all osteosarcomas. ${ }^{2,13}$ They tend to occur in an older age group usually between 30 and 39 years and have an overall better prognosis than conventional osteosarcoma. ${ }^{13,40,41}$ Risk factors include prior
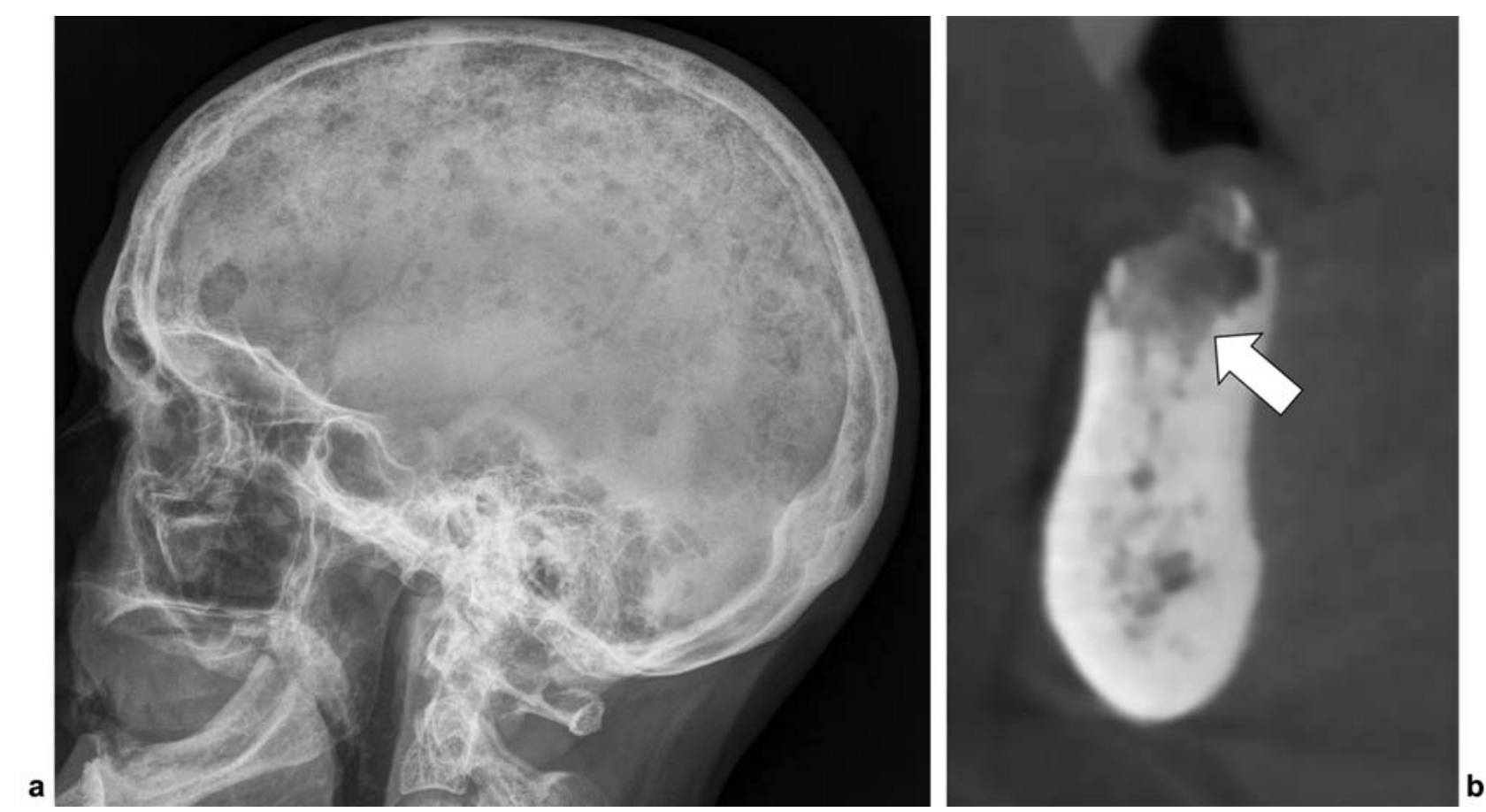

Fig. 13 Bisphosphonate-related osteonecrosis of the jaws. (a) Lateral view of the skull shows multiple punched-out lesions in a patient with multiple myeloma that was therefore treated by bisphosphonates. (b) Sagittal reformatted CBCT image shows an ill-defined area of mixed density in the right mandible (arrow). 


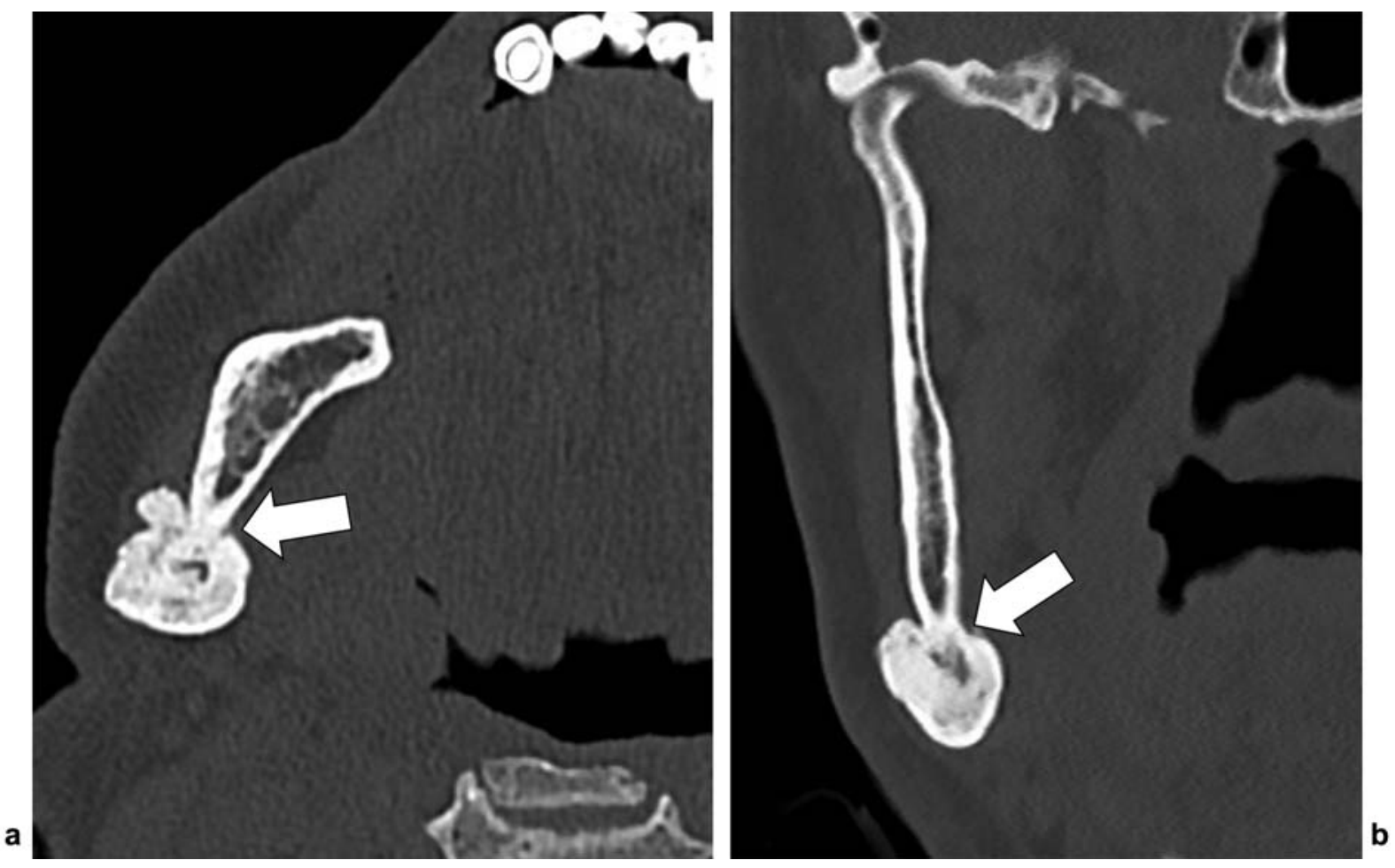

Fig. 14 Osteochondroma of the mandible. (a) Axial and (b) coronal CT image shows a mushroom-shaped radiopaque lesion at the right mandibular angle. Note continuity of the cortical and trabecular bone of the lesion and the mandible (arrows).

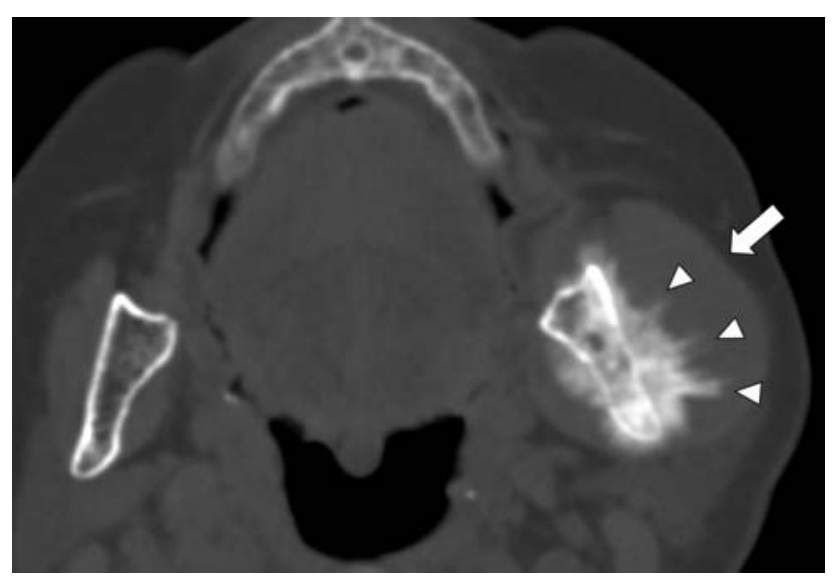

Fig. 15 Osteoblastic metastasis of a breast carcinoma. Axial CT shows an osteoblastic bone lesion in the left mandible with aggressive hair-on-end periosteal reaction (arrowheads) and soft tissue extension (arrow). This type of periosteal reaction may also be seen in a gnathic osteosarcoma.

radiation therapy of the head and neck region and Paget's disease of bone. ${ }^{13}$

Early stage gnathic osteosarcoma may be osteolytic, causing widening of the periodontal ligament or irregular and indistinct lining of the mandibular canal. ${ }^{2,15}$ Osteolytic lesions may cause bone resorption around the roots of teeth, causing a floating or hanging tooth appearance. ${ }^{2}$ More advanced lesions (-Fig. 16) are typically osteoblastic or most commonly mixed on radiographs and CBCT, due to the presence of mineralized osteoid matrix. ${ }^{2}$

These lesions are typically destructive with poorly defined margins, cortex destruction, and aggressive periosteal reaction (hair-on-end or sunray type) in $25 \%$ of cases. ${ }^{2,13}$ CBCT is the

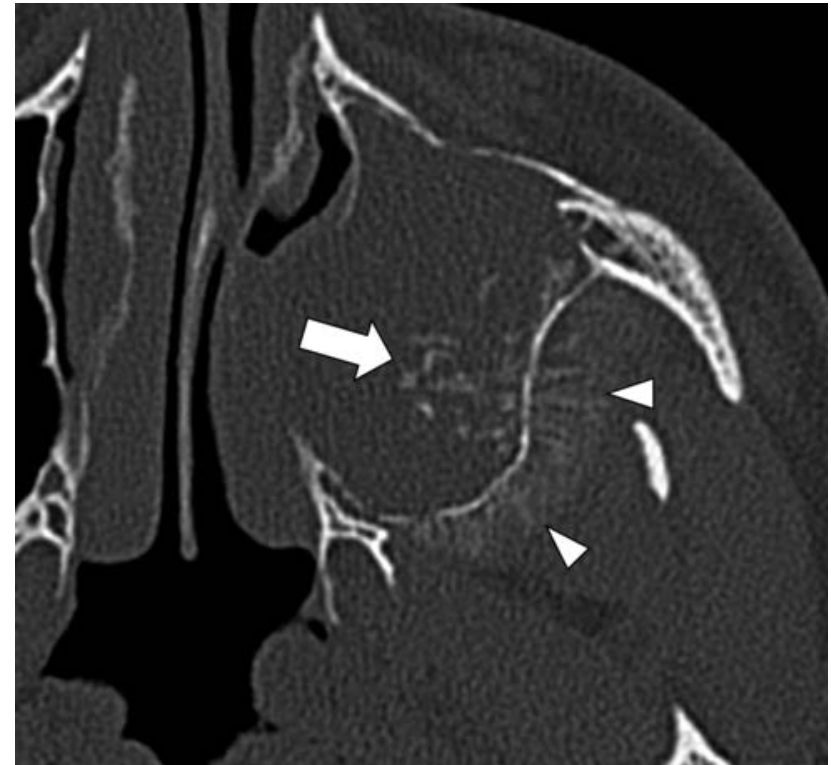

Fig. 16 Osteosarcoma of the left maxillary sinus. Axial dental CT shows opacification of the left maxillary sinus with intralesional calcification (arrow) and hair-on-end periosteal reaction in the left fossa infratemporalis (arrowheads). Reprinted with permission from JBSR.

preferred modality to detect the presence of osteoid matrix, whereas MRI is the modality of choice for local staging, evaluation of bone marrow, and soft tissue involvement ( - Fig. S10). ${ }^{40}$

\section{Odontogenic Myxoma/Myxofibroma}

Odontogenic myxoma/myxofibromas are rare odontogenic lesions occurring in the second to fourth decade of life. ${ }^{15}$ The lesions are benign but may be locally aggressive causing 


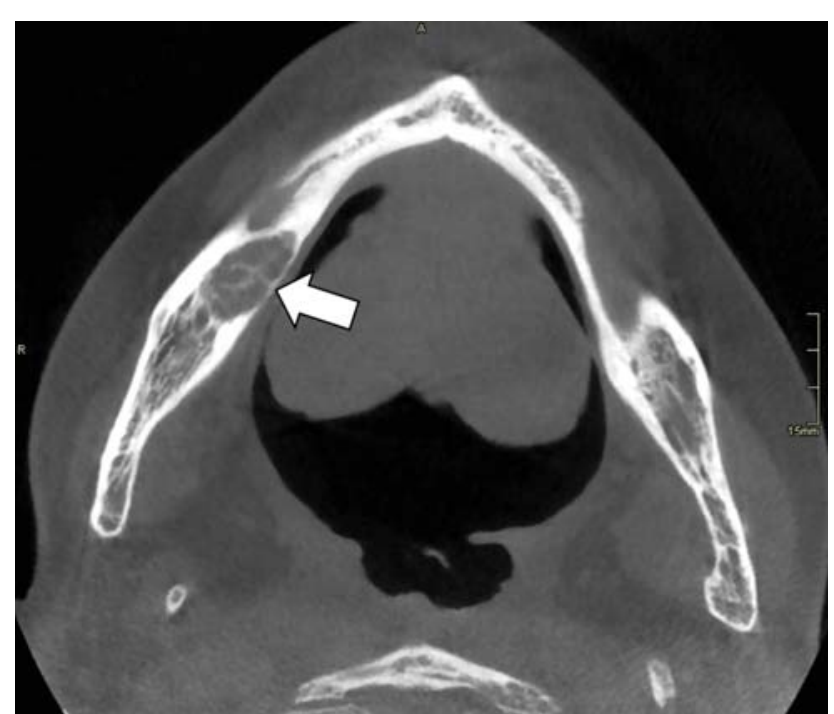

Fig. 17 Odontogenic myxoma. Axial CBCT shows an osteolytic lesion of the right mandible with intralesional trabeculations resulting in a honeycomb appearance (arrow).

cortical destruction and resorption of adjacent teeth on radiographs and CBCT. Lesions are well defined or ill-defined and may be unilocular or multilocular. Intralesional trabeculations may result in a honeycomb or tennis-racket appearance on radiographs (-Fig. 17). The lesions may have a mixed density due to irregular intralesional calcifications. ${ }^{17}$

\section{Osteoid Osteoma and Osteoblastoma}

Although osteoid osteoma is a common benign bone tumor, it only rarely affects the jawbones, with the posterior mandible the most frequently involved. It is often painful, most pronounced at night, and mitigated by treatment with salicylates.

On radiographs and $\mathrm{CBCT}$, the lesion shows a radiolucent nidus (often with a central calcification), surrounded by extensive sclerosis. ${ }^{42}$ On MRI, the lesion is surrounded by extensive bone and soft tissue edema, but the small nidus is often less visible than on CBCT.

Osteoblastoma is regarded as a giant osteoid osteoma. It also rarely affects the craniofacial bones, with a similar predilection as osteoid osteoma for the posterior mandible. It affects young patients with a mean age $<20$ years.

Radiologically, the lesion is usually ill-defined and radiolucent or of mixed opacity, often with sclerotic margins and intralesional calcifications. ${ }^{33}$ On MRI, the lesion shows marked enhancement. ${ }^{33}$

\section{Calcifying (Epithelial) Odontogenic Cyst}

The calcifying odontogenic cyst ( $\mathbf{- F i g . ~ S 1 1 ) ~ i s ~ a l s o ~ k n o w n ~ a s ~}$ keratinizing and calcifying ameloblastoma, keratinizing and calcifying odontogenic cyst, or Gorlin cyst. ${ }^{43}$ The lesion may present either as a unilocular radiolucency or as a lesion with a mixed radiolucent/radiopaque appearance. ${ }^{44}$

\section{Fibrous Dysplasia and Paget's Disease}

Fibrous dysplasia and Paget's disease are discussed further in the article "Fibrous Dysplasia, Paget's Disease, and Uncommon Bone Lesions of the Craniofacial Bones" in this issue.

\section{Conclusion}

The combination of clinical presentation, age, lesion distribution, and imaging findings are the cornerstones for appropriate lesion characterization of most radiopaque lesions and lesions of mixed density of the jaws. This is pivotal for defining further lesion management. Indeterminate lesions should be referred for biopsy and subsequent histopathologic examination. It is of utmost importance that interpretation of histopathologic specimens be correlated with clinical and radiologic examinations to obtain a correct definitive diagnosis.

Conflict of Interest

None declared.

Acknowledgments

We thank Johan Hintjens, MD, Didier Dielen, MD, and Liesbeth Verschaeren for their help in the preparation of this article.

\section{References}

1 Dunfee BL, Sakai O, Pistey R, Gohel A. Radiologic and pathologic characteristics of benign and malignant lesions of the mandible. Radiographics 2006;26(06):1751-1768

2 Bernaerts A, Vanhoenacker FM, Hintjens J, et al. Tumors and tumor-like lesions of the jaw mixed and radiopaque lesions. JBR-BTR 2006;89(02):91-99

3 Harmon M, Arrigan M, Toner M, O'Keeffe SA. A radiological approach to benign and malignant lesions of the mandible. Clin Radiol 2015;70(04):335-350

4 Curé JK, Vattoth S, Shah R. Radiopaque jaw lesions: an approach to the differential diagnosis. Radiographics 2012;32(07):1909-1925

5 More C, Thakkar K, Asrani M. Cemento-ossifying fibroma. Indian J Dent Res 2011;22(02):352-355

6 Trijolet J-P, Parmentier J, Sury F, Goga D, Mejean N, Laure B. Cemento-ossifying fibroma of the mandible. Eur Ann Otorhinolaryngol Head Neck Dis 2011;128(01):30-33

7 Liu Y, Wang H, You M, et al. Ossifying fibromas of the jaw bone: 20 cases. Dentomaxillofac Radiol 2010;39(01):57-63

8 MacDonald-Jankowski DS. Ossifying fibroma: a systematic review. Dentomaxillofac Radiol 2009;38(08):495-513

9 Sontakke SA, Karjodkar FR, Umarji HR. Computed tomographic features of fibrous dysplasia of maxillofacial region. Imaging Sci Dent 2011;41(01):23-28

10 Weber AL. Imaging of cysts and odontogenic tumors of the jaw. Definition and classification. Radiol Clin North Am 1993;31(01): 101-120

11 Fenerty S, Shaw W, Verma R, et al. Florid cemento-osseous dysplasia: review of an uncommon fibro-osseous lesion of the jaw with important clinical implications. Skeletal Radiol 2017;46(05):581-590

12 Gonçalves M, Píspico R, Alves Fde A, Lugão CEB, Gonçalves A. Clinical, radiographic, biochemical and histological findings of florid cemento-osseous dysplasia and report of a case. Braz Dent $J$ 2005;16(03):247-250

13 Eldaya R, Eissa O, Herrmann S, Pham J, Calle S, Ribe T. Mandibular lesions: a practical approach for diagnosis based on multimodality imaging findings. Contemp Diagnost Radiol 2017;40(06):1-8

14 Singer SR, Mupparapu M, Rinaggio J. Clinical and radiographic features of chronic monostotic fibrous dysplasia of the mandible. J Can Dent Assoc 2004;70(08):548-552

15 Neyaz Z, Gadodia A, Gamanagatti S, Mukhopadhyay S. Radiographical approach to jaw lesions. Singapore Med J 2008;49(02): 165-176; quiz 177 
16 de Oliveira BH, Campos V, Marçal S. Compound odontomadiagnosis and treatment: three case reports. Pediatr Dent 2001; 23(02):151-157

17 Chapman MN, Nadgir RN, Akman AS, et al. Periapical lucency around the tooth: radiologic evaluation and differential diagnosis. Radiographics 2013;33(01):E15-E32

18 Eversole R, Su L, ElMofty S. Benign fibro-osseous lesions of the craniofacial complex. A review. Head Neck Pathol 2008;2(03):177-202

19 Scholl RJ, Kellett HM, Neumann DP, Lurie AG. Cysts and cystic lesions of the mandible: clinical and radiologic-histopathologic review. Radiographics 1999;19(05):1107-1124

20 Gupta M, Kaste SC, Hopkins KP. Radiologic appearance of primary jaw lesions in children. Pediatr Radiol 2002;32(03):153-168

21 Wright JM, Soluk Tekkeşin M. Odontogenic tumors: where are we in 2017? J Istanb Univ Fac Dent 2017;51(03, Suppl 1):S10-S30

22 Kaneda T, Minami M, Kurabayashi T. Benign odontogenic tumors of the mandible and maxilla. Neuroimaging Clin N Am 2003;13 (03):495-507

23 Kaplan I, Buchner A, Calderon S, Kaffe I. Radiological and clinical features of calcifying epithelial odontogenic tumour. Dentomaxillofac Radiol 2001;30(01):22-28

24 More CB, Vijayvargiya R. Intraosseous calcifying epithelial odontogenic (Pindborg) tumor: A rare entity. J Oral Maxillofac Pathol 2015;19(02):269

25 Garcia Garcia B, Ruiz Masera JJ, Zafra Camacho FM, Centella Gutierrez C. Intraosseous dentinogenic ghost cell tumor: Case report and treatment review. Rev Esp Cir Oral Maxilofac 2015;37 (04):243-246

26 Li BB, Gao Y. Ghost cell odontogenic carcinoma transformed from a dentinogenic ghost cell tumor of maxilla after multiple recurrences. Oral Surg Oral Med Oral Pathol Oral Radiol Endod 2009; 107(05):691-695

27 Agrawal Y, Naidu GS, Makkad RS, et al. Dentinogenic ghost cell tumor-a rare case report with review of literature. Quant Imaging Med Surg 2017;7(05):598-604

28 Hoff AO, Toth B, Hu M, Hortobagyi GN, Gagel RF. Epidemiology and risk factors for osteonecrosis of the jaw in cancer patients. Ann N Y Acad Sci 2011;1218(01):47-54

29 Özgür A, Kara E, Arpacı R, et al. Nonodontogenic mandibular lesions: differentiation based on CT attenuation. Diagn Interv Radiol 2014;20(06):475-480
30 Deshpande SS, Thakur MH, Dholam K, Mahajan A, Arya S, Juvekar S. Osteoradionecrosis of the mandible: through a radiologist's eyes. Clin Radiol 2015;70(02):197-205

31 Hermans R, Fossion E, Ioannides C, Van den Bogaert W, Ghekiere J, Baert AL. CT findings in osteoradionecrosis of the mandible. Skeletal Radiol 1996;25(01):31-36

32 Hermans R. Imaging of mandibular osteoradionecrosis. Neuroimaging Clin N Am 2003;13(03):597-604

33 Avril L, Lombardi T, Ailianou A, et al. Radiolucent lesions of the mandible: a pattern-based approach to diagnosis. Insights Imaging 2014;5(01):85-101

34 Tsuchimochi M, Kurabayashi T. Symposium: Imaging modalities for drug-related osteonecrosis of the jaw (1), role of imaging in drug-related osteonecrosis of the jaw: an up-to-date review (secondary publication). Jpn Dent Sci Rev 2019;55(01):1-4

35 Mohan Choontharu M, Ahmad Buch S, Subhas Babu G, et al. A rare clinical presentation of an osteochondroma of coronoid process of mandible. J Dent (Shiraz) 2018;19(04):325-330

36 Navaneetham A, Rao KA, Kumaran S, Baweja HH. A unique case of multiple osteochondroma: mandibular symphysis and femur. Ann Maxillofac Surg 2012;2(02):182-184

37 Vanhoenacker FM, Van Hul W, Wuyts W, Willems PJ, De Schepper AM. Hereditary multiple exostoses: from genetics to clinical syndrome and complications. Eur J Radiol 2001;40(03):208-217

38 Kumar G, Manjunatha B. Metastatic tumors to the jaws and oral cavity. J Oral Maxillofac Pathol 2013;17(01):71-75

39 Muttagi SS, Chaturvedi P, D'Cruz A, et al. Metastatic tumors to the jaw bones: retrospective analysis from an Indian tertiary referral center. Indian J Cancer 2011;48(02):234-239

40 Theodorou DJ, Theodorou SJ, Sartoris DJ. Primary non-odontogenic tumors of the jawbones: an overview of essential radiographic findings. Clin Imaging 2003;27(01):59-70

41 Vanhoenacker FM, Camerlinck M, De Vuyst D, Chapelle K. Osteosarcoma of the maxilla. JBR-BTR 2012;95(03):168-169

42 Singh A, Solomon MC. Osteoid osteoma of the mandible: a case report with review of the literature. J Dent Sci 2017;12(02):185-189

43 Uchiyama Y, Akiyama H, Murakami S, et al. Calcifying cystic odontogenic tumour: CT imaging. Br J Radiol 2012;85(1013):548-554

44 Sonone A, Sabane VS, Desai R. Calcifying ghost cell odontogenic cyst: report of a case and review of literature. Case Rep Dent 2011; 2011:328743 